\title{
Abnormal mixing of passive scalars in chaotic flows
}

\author{
O. V. Popovych, ${ }^{1,2}$ A. Pikovsky, ${ }^{1}$ and B. Eckhardt ${ }^{3}$ \\ ${ }^{1}$ Department of Physics, University of Potsdam, 14415 Potsdam, Germany \\ ${ }^{2}$ Institute of Medicine, Research Center Jülich, 52425 Jülich, Germany \\ ${ }^{3}$ Department of Physics, Philipps-University of Marburg, 35032 Marburg, Germany
}

(Received 8 June 2006; published 19 March 2007)

\begin{abstract}
We study the relaxation of a passive scalar towards the uniform equilibrium distribution in an advectiondiffusion problem where the phase space for the pure advection problem is a mixture of chaotic domains and elliptic islands. Since the advection-diffusion problem is linear, the relaxation can be characterized by the eigenvalues and eigenmodes of the evolution operator. Almost degenerate eigenvalues then give rise to deviations from simple exponential decay behavior. We show by example that the corresponding eigenmodes can be supported by islands or weakly connected chaotic domains. These theoretical considerations are related to some experimental observations in two-dimensional flows.
\end{abstract}

DOI: 10.1103/PhysRevE.75.036308

Stirring by chaotic advection greatly enhances the mixing of tracers in a fluid. The dynamical stretching and folding mechanism of chaotic flows produces intricate patterns, down to ever finer detail, until eventually diffusion takes over and completes the mixing on the molecular level [1-6]. This mechanism is particularly attractive for applications in low-Reynolds-number situations such as high-viscosity fluids or microchannels [7-10]. The dynamics of this stirring process has been probed in some detail in recent experiments on two-dimensional (2D) flows [11-14]. In addition to observations of the persistent patterns predicted by Pierrehumbert [15] the experiments also analyzed the decay rate of the contrast in the pattern. The evolution of a passive scalar field is governed by linear advection-diffusion equations, which allow an analysis in terms of eigenvalues and eigenfunctions which suggests exponential decays, perhaps modulated by oscillations if the eigenvalues are complex. The nonexponential decay observed in certain experiments in [13] was argued to be due to the large spatial extension and thus the coalescence of many eigenvalues. The goal of this paper is to show that also in small systems deviations from an exponential law can be found if the phase space is inhomogeneous: the decay can be dominated by several nearly degenerate eigenvalues, and no simple exponential decay results. We will also describe dynamical mechanisms for such a behavior. As a consequence, techniques for the destruction of islands of quasiintegrable motion, such as the resonance effects discussed in [9], will be very important for the design of efficient micromixers $[8,10]$.

The theoretical description of the experiments can be based on a linear advection-diffusion equation for a passive scalar density $\phi(x, y, t)$,

$$
\frac{\partial \phi}{\partial t}+\boldsymbol{v}(\boldsymbol{x}, t) \cdot \nabla \phi=D \Delta \phi
$$

where $\boldsymbol{v}(x, y, t)$ is the flow velocity field (assumed to be incompressible) and $D$ is a molecular diffusion coefficient. The linearity of Eq. (1) and the time periodicity of the velocity field $\boldsymbol{v}(x, y, t)$ suggest an expansion of the density in a basis given by the Floquet eigenmodes of the linear evolution op-
PACS number(s): 47.52. $+\mathrm{j}, 05.40 . \mathrm{Jc}$

erator $[15,16]$. There will be one eigenvalue equal to 1 , with the eigenvector the invariant density, and the one closest to it, with modulus less than 1 , will dominate the asymptotic

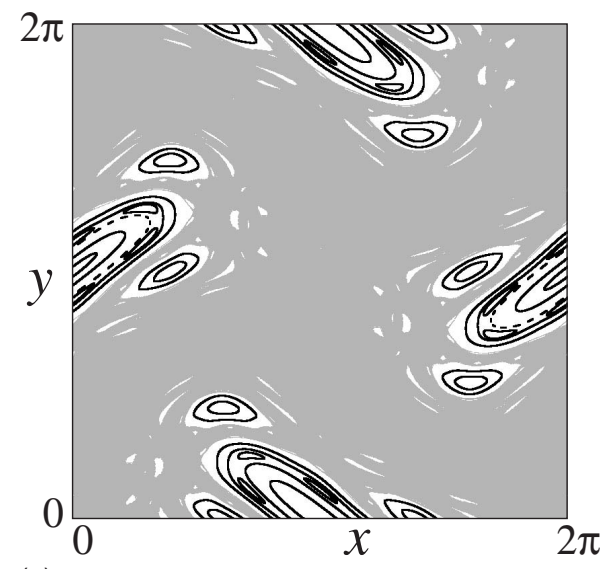

(a)

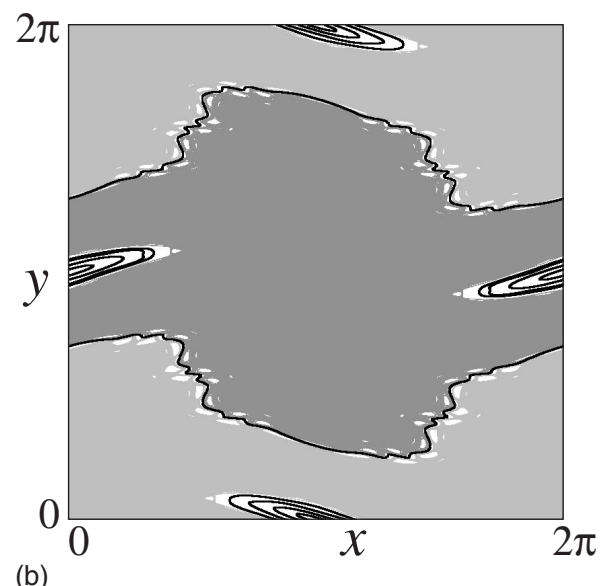

FIG. 1. Phase portraits of the map (4). A long trajectory that fills out a chaotic region and leaves blank the elliptic islands is shown in gray. A few individual trajectories with quasiperiodic dynamics are shown in black. Parameters are $U_{1}=5$ and $U_{2}=3$ in (a) and $U_{1}$ $=12$ and $U_{2}=1.12$ for (b). Note that in (b) there are two separate chaotic regions, distinguished by lighter and darker shades of gray. 


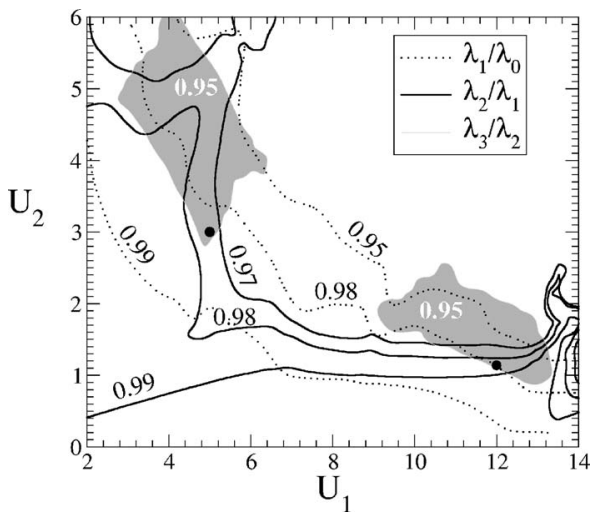

FIG. 2. Contour lines for the two largest eigenvalues as functions of the flow parameters $U_{1}$ and $U_{2}$. The region where the ratio $\lambda_{3} / \lambda_{2}$ is larger than 0.95 is depicted by gray. Two particular parameter sets discussed in details are depicted with black circles.

dynamics for long times: the contrast of the scalar distribution, defined as

$$
\delta \phi^{2}=\left\langle(\phi-\langle\phi\rangle)^{2}\right\rangle,
$$

will decay exponentially with the square of the second largest eigenvalue of the corresponding transfer operator [17-23].

The detailed description of the experimental flow is somewhat involved [24], and simulations are not very efficient
TABLE I. The four largest eigenvalues of the Frobenius-Perron operator (5) of the noisy map (4) with $D T=0.005$.

\begin{tabular}{lrccc}
\hline \hline$U_{1}, U_{2}$ & $\lambda_{0}$ & $\lambda_{1}$ & $\lambda_{2}$ & $\lambda_{3}$ \\
\hline$U_{1}=5, U_{2}=3$ & 1.0000 & 0.9890 & 0.9743 & 0.9373 \\
$U_{1}=12, U_{2}=1.14$ & 1.0000 & 0.9865 & 0.9719 & 0.9344 \\
\hline \hline
\end{tabular}

because of the full time dependence. However, the same qualitative features can be obtained in flows that reduce to 2D-area-preserving maps [25]. Consider the family of flows

$$
\boldsymbol{v}(x, y, t)=\left(\begin{array}{l}
U_{1} f_{1}(t) \sin (y) \\
U_{2} f_{2}(t) \sin (x)
\end{array}\right)
$$

on a domain periodic in $x$ and $y$ with period $2 \pi$. The timedependent functions $f_{1}(t)$ and $f_{2}(t)$ are periodic with period $T$ and describe the switching between two simple shear flows,

$$
f_{1}(t)=1-f_{2}(t)= \begin{cases}1 & \text { if } 0 \leqslant t<T / 2, \\ 0 & \text { if } T / 2 \leqslant t<T,\end{cases}
$$

of amplitudes $U_{1}$ and $U_{2}$. The equations of motion can easily be integrated, so that the evolution over one period can be described by the two-dimensional map

$$
x_{n+1}=x_{n}+\frac{1}{2} U_{1} \sin \left(y_{n}\right) \quad(\bmod 2 \pi),
$$

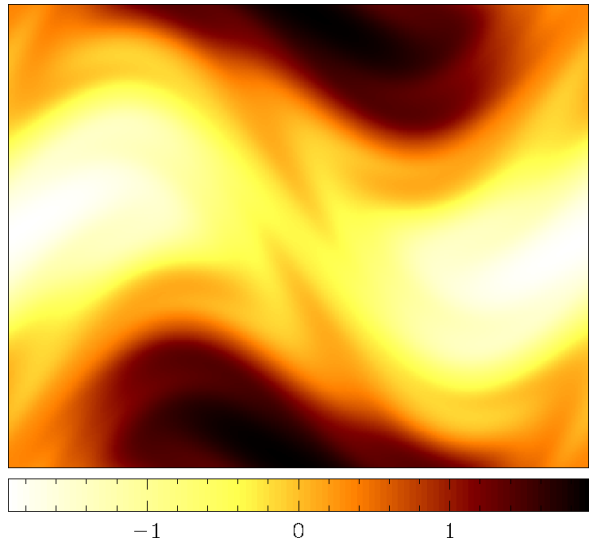

(a)

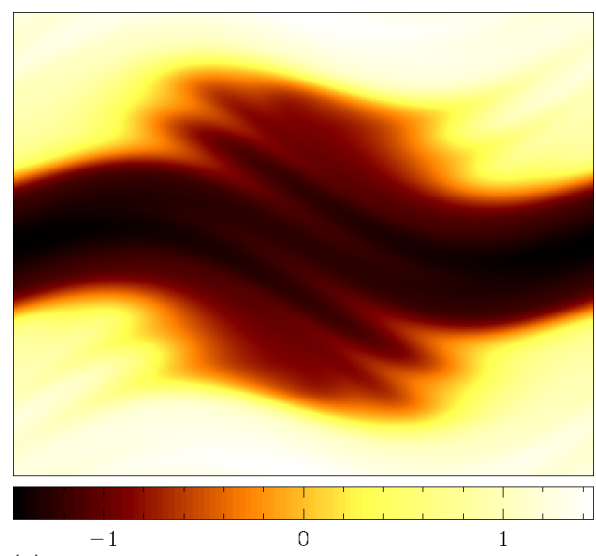

(c)

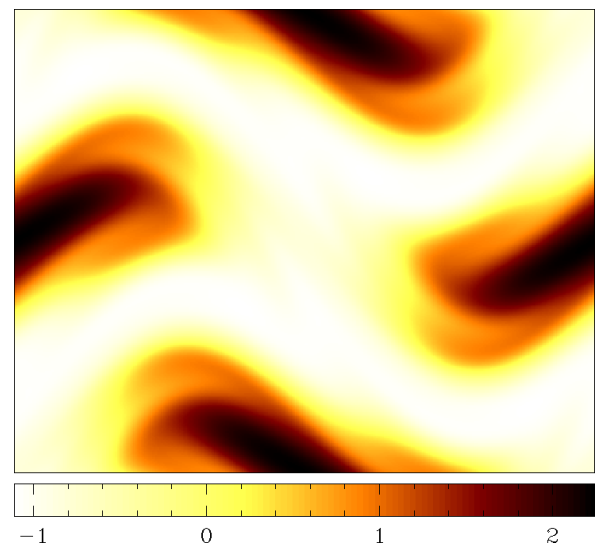

(b)

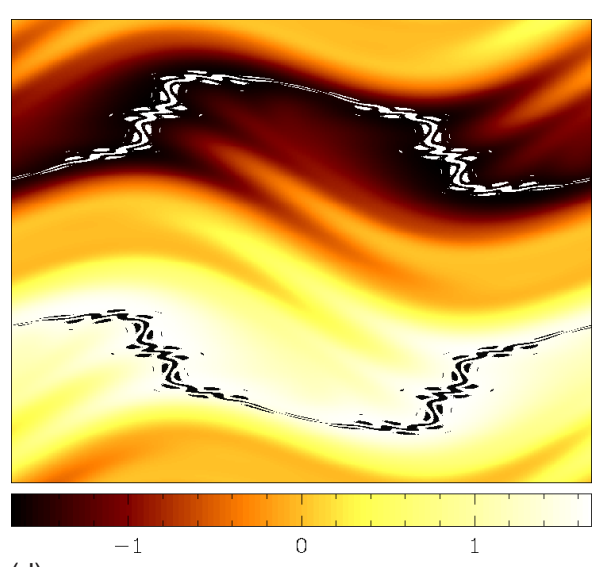

(d)
FIG. 3. (Color online) Eigendensities for the Frobenius-Perron operator (5). The panels in the top row $[(a)$ and (b)] are for the first parameter set in Table I, and the ones in the bottom row are for the second parameter set. The eigenvalues are $\lambda_{1}$ in (a) and (c) and $\lambda_{2}$ in (b) and (d). In (d) the border between two chaotic regions shown in Fig. 1(b) for parameters $U_{1}=12$ and $U_{2}=1.12$ is shown in black and white. 


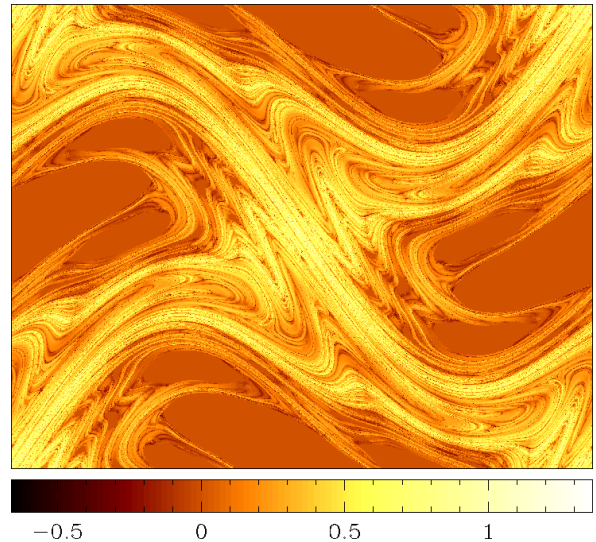

(a)

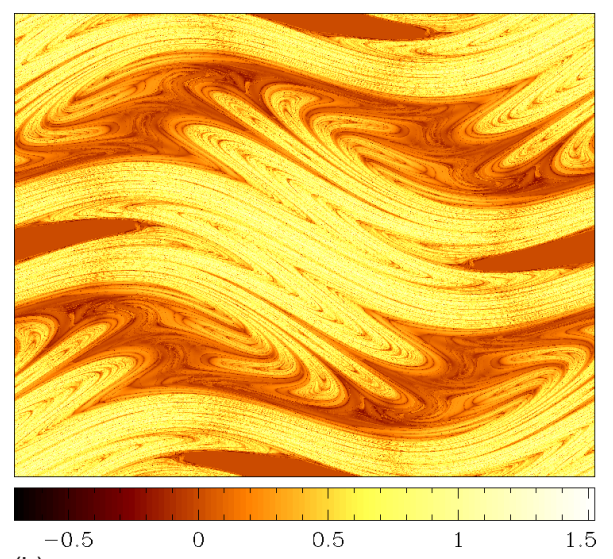

(b)

FIG. 4. (Color online) Local Lyapunov exponent for the map (4) averaged over ten iterations. Parameters (a) $U_{1}=5$ and $U_{2}=3$, (b) $U_{1}=12$ and $U_{2}=1.14$.

$$
y_{n+1}=y_{n}+\frac{1}{2} U_{2} \sin \left(x_{n+1}\right) \quad(\bmod 2 \pi)
$$

(here $n$ is discrete time measured in units of $T$ ). The phasespace dynamics of the map (4) for two sets of parameters is shown in Fig. 1.

In order to simplify further the advection-diffusion equation (1), we consider a model situation where the actions of the advection and diffusion are separated in time as well (cf. [18]). We assume that within each period $T$ the scalar field first evolves according to Eqs. (1) and (3) with $D=0$ and then is subjected to a diffusional spreading according to Eq. (1) with $\boldsymbol{v}=0$. The action of these processes on a scalar density can be described by the Frobenius-Perron operator (FPO) $\mathcal{F}_{A}$ for the map (4) and the operator $\mathcal{F}_{D}=\exp (t D \Delta)$ for free diffusion over a time $T$. The full propagator for one time step then is their operator product, $\mathcal{F}=\mathcal{F}_{D} \circ \mathcal{F}_{A}$. For the numerical simulation of time evolutions it is worth noting that the same equation describes the evolution of the probability density of Eqs. (4) with additive white Gaussian noise of strength $\sigma^{2}$ $=2 D T$.

The periodicity of the domain suggests to use a Fourier representation for both operators (cf. $[18,26]$ ). The Fourier harmonics of the scalar density,

$$
\psi_{n}(l, m)=\frac{1}{4 \pi^{2}} \iint d x d y \phi(x, y, n) e^{-i(l x+m y)},
$$

are then mapped according to

$$
\begin{aligned}
\psi_{n+1}(l, m)= & \exp \left[-\left(l^{2}+m^{2}\right) D T\right] \\
& \times \sum_{i, j} \psi_{n}(i, j) J_{j-m}\left(\frac{i U_{1}}{2}\right) J_{i-l}\left(\frac{m U_{2}}{2}\right),
\end{aligned}
$$

with $J_{k}(s)$ Bessel functions of the first kind.

The usual approach to a study of the spectral properties of the linear infinite-dimensional Frobenius-Perron operator (5) is to cut off the number of Fourier harmonics $\left\{\psi_{n}(l, m)\right\}$ at some large value (depending on the parameter $D T$ ) and to deal thereafter with finite matrices only. Since the matrix elements decay exponentially with increasing indices $l$ and $m$, we take $-50 \leqslant l, m \leqslant 50$ for the case $D T=0.005$ studied here. Then the matrix elements for $l, m= \pm 50$ are at most of order $10^{-6}$ and a further increase of the matrix dimension does not result in significant changes to the largest eigenvalues. These eigenvalues depend on the parameters of the flow, $U_{1}$ and $U_{2}$, as illustrated in Fig. 2. Since we are looking for degenerate eigenvalues, we show the ratio between the nextto-leading eigenvalues $\lambda_{1}$ and the leading eigenvalues $\lambda_{0}$ and the one between $\lambda_{2}$ and $\lambda_{1}$ by contour lines and the ratio $\lambda_{3} / \lambda_{1}$ by gray shading. The figure shows that these ratios vary in a rather unpredictable manner with the parameters $U_{1}$ and $U_{2}$, which can be attributed to the fine structures in the phase spaces shown in Fig. 1. For the two parameter values indicated by black dots, all three ratios are above 0.95 , and the further discussion shows that nonexponential decay does occur.

For a further discussion we pick the two characteristic parameter pairs $\left(U_{1}=5, U_{2}=3\right)$ and $\left(U_{1}=12, U_{2}=1.14\right)$, indicated in Fig. 2. The phase-space structure for the first pair is shown in Fig. 1(a). The parameter values for the second pair are slightly shifted from those in Fig. 1(b): for $U_{2}=1.12$ the two chaotic regions shown in Fig. 1(b) are separate, whereas for $U_{2}=1.14$ they have merged. Table I gives the four largest eigenvalues obtained by this procedure for these two sets of parameters.

Table I confirms that there is an isolated unit eigenvalue $\lambda_{0}$ corresponding to the invariant homogeneous distribution. The distance between the second and third largest eigenvalues $\lambda_{1}$ and $\lambda_{2}$ is about 0.015 and much smaller than the distance between $\lambda_{2}$ and $\lambda_{3}$ (which is about 0.037). The eigenfunctions for $\lambda_{1}$ and $\lambda_{2}$ are shown in Fig. 3 .

We note that in order to have degenerate eigenvalues $\lambda_{1}=\lambda_{2}$ the parameters $U_{1}$ and $U_{2}$ have to be adjusted properly. On the other hand, a noticeable effect on the decay of the contrast results already if the eigenvalue difference is of the same order as their distance from $\lambda_{0}=1$ : this leaves a significant region of parameters with close eigenvalues.

The two sets of parameters in Table I and Fig. 3 are qualitatively different from the point of view of the structure of the eigenmodes. In the first case $\left(U_{1}=5\right.$ and $\left.U_{2}=3\right)$ there are two big elliptic islands [Fig. 1(a)] and the slowest eigenmodes are located in these regions [Figs. 3(a) and 3(b)]. For the second set of parameters $\left(U_{1}=12\right.$ and $\left.U_{2}=1.14\right)$, any 


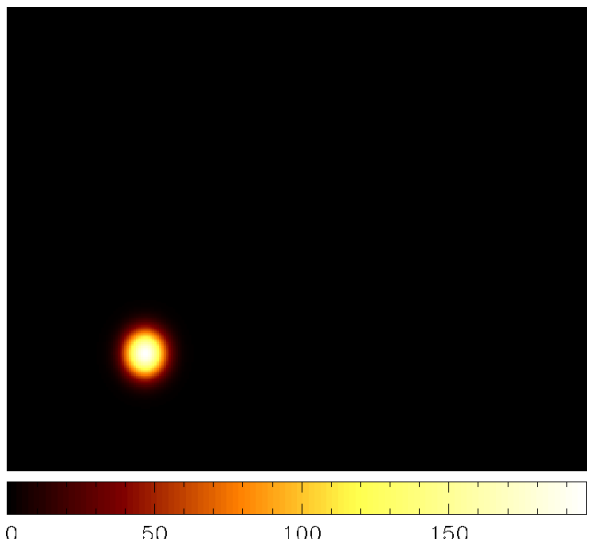

(a)

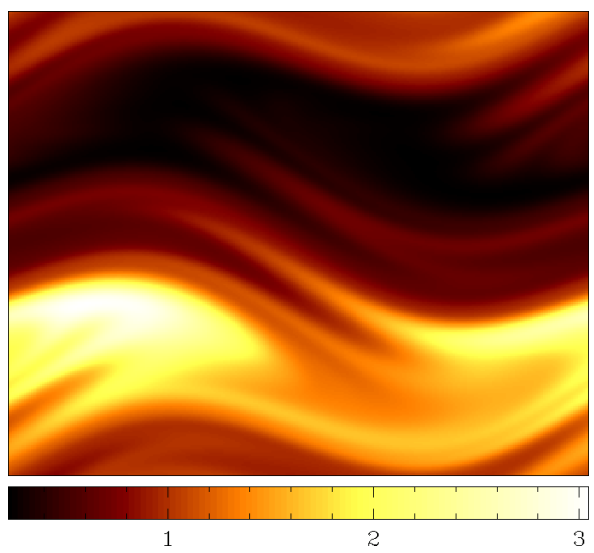

(b)

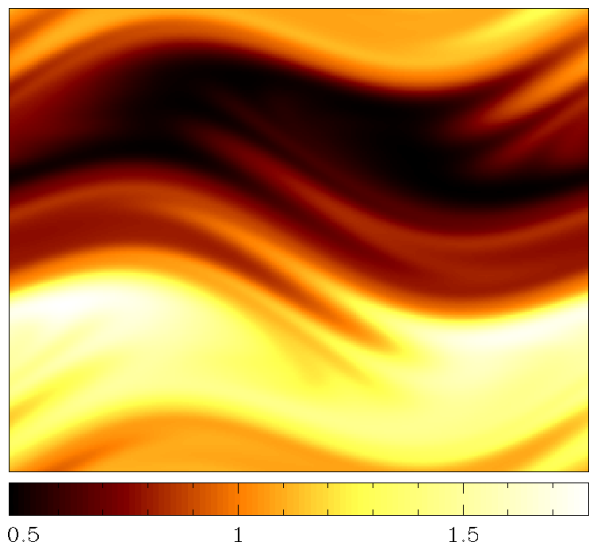

(c)

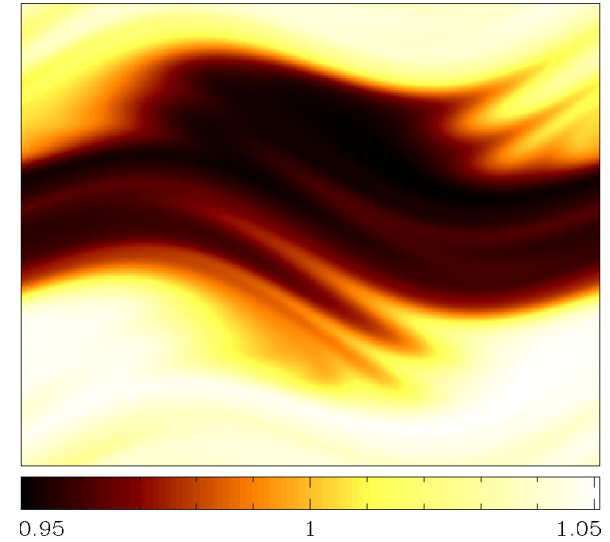

(d)

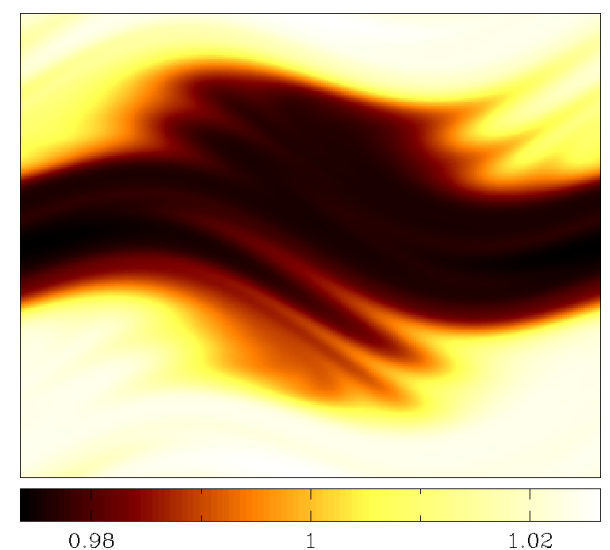

(e)

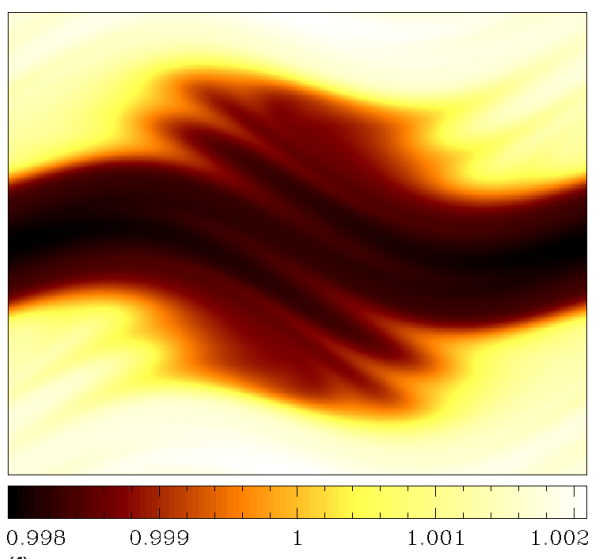

(f)
FIG. 5. (Color online) Evolution of the scalar $\phi(x, y, t)$ advected by flow (3) with parameters $U_{1}=12, U_{2}=1.14$, and $D T$ $=0.005$. The initial distribution (a) is a Gaussian with mean $(1.5 ; 1.6)$ and standard deviation 0.15 . The times for the states of $\phi(x, y, t)$ shown in the other frames are (b) $t=40 T$, (c) $t=60 T$, (d) $t=160 T$, (e) $t=200 T$, and (f) $t=400 T$. elliptic islands that might be present are small [Fig. 1(b)] and the eigenmodes are not restricted to them [Figs. 3(c) and 3(d)]. However, for these parameter values there are two chaotic regions in phase space that are either isolated [two coexisting invariant chaotic regions as in Fig. 1(b)] or have a low probability of transition between them [as is the case for the parameters used for Figs. 3(c) and 3(d) for Fig. 1(b) we have $U_{2}=1.12$, and for the eigendensities in Fig. 3, $U_{2}$ $=1.14$; this small difference is enough to connect the two chaotic regions weakly]. This additional slow dynamics of the transitions between the two chaotic regions leads to an eigenvalue of the FPO close to unity. On the other hand, the mixing in the boundary layers of the two chaotic regions is very weak and this again results in a large eigenvalue. The slowest eigenmodes are thus supported by these two chaotic domains and their boundary [Figs. 3(c) and 3(d)].

To support this idea, we have calculated local Lyapunov exponents [27] for the map (4) and plotted them in Fig. 4. They have been averaged over ten iterations and assigned to the last state point in the sequence. One can see from Fig. 4 that the spatial structure of the calculated exponents is a replica of the slow eigenmodes presented in Fig. 3. In the domains with small local exponents the mixing is slow. The regions with small local exponents that stand out in Fig. 4 are elliptic islands and a boundary between chaotic regions (cf. Figs. 4 and 1). 

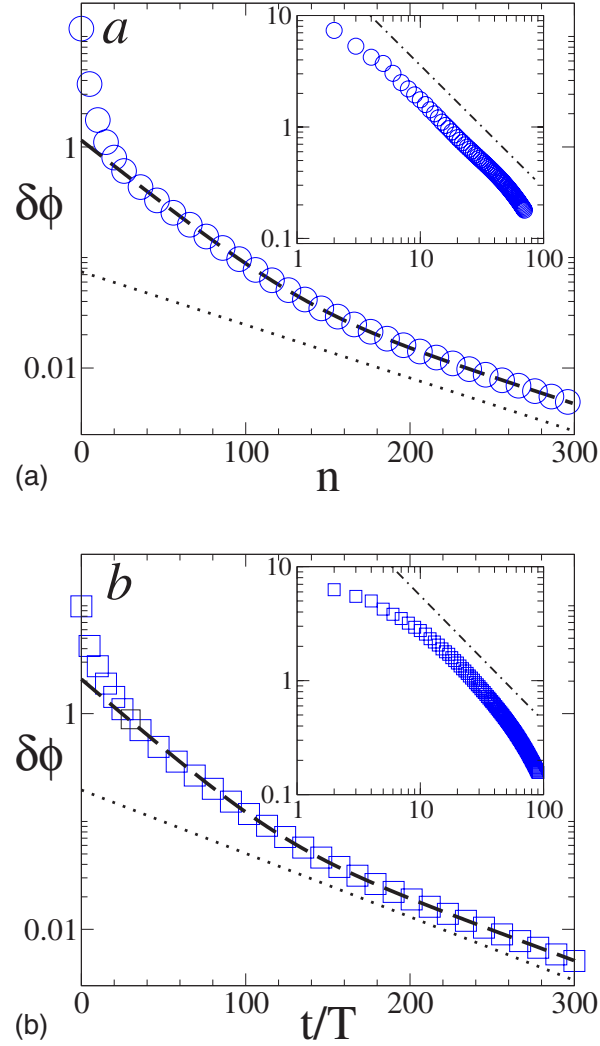

FIG. 6. (Color online) Linear-logarithmic plot of the decay of contrast $\delta \phi$ as a function of time for the parameter values given in Table I. The symbols are from numerical solutions of the advectiondiffusion equation. The bold dashed curves depict the theoretical prediction of the decay according to (6) with fitted parameters $b_{i}$ and $\lambda_{i}^{\prime}$ [in case (a), $\lambda_{1}^{\prime}=0.9890, \lambda_{2}^{\prime}=0.9734$; in case (b), $\lambda_{1}^{\prime}$ $\left.=0.9871, \lambda_{2}^{\prime}=0.9703\right]$. For comparison, the dotted lines have the slopes of the asymptotically dominant eigenvalue $\lambda_{1}$ from Table I. In the insets the contrast $\delta \phi$ is shown on a double-logarithmic scale. The dot-dashed lines there have the slope -1.1 . For the solution of the advection-diffusion equations an initial distribution of the density $\phi(x, y, 0)$ taken in a Gaussian form has been simulated in two different ways: (i) for the discrete noisy map (4) with the help of its FP operator (5) for $D T=0.005$ and (ii) for the continuous-time system (1) and (3) for $D=0.005$ and $T=1$. Panel (a) was computed using (i) and panel (b) using (ii). The agreement with formula (6) shows that both representations give results of similar quality.

In order to study the evolution of the passive scalar $\phi(x, y, t)$ in the full equations (1) and (3) we use a Fourier representation and integrate numerically the system of ordinary differential equations for the $301 \times 301$ largest Fourier modes [25]. The results of this direct numerical integration shown in Fig. 5 are for the second set of parameters $U_{1}$ $=12$ and $U_{2}=1.14$.

For suitable initial conditions one observes a competition between different patterns: after a few periods of forcing a spatial structure dominated by the two next to leading eigenmodes of Figs. 3(c) and 3(d) is observed. Beginning with frame (b) of Fig. 5 these patterns can be seen for some time [Figs. 5(c) and 5(d)], but eventually only the slowest eigenmode (corresponding to the second largest eigenvalue $\lambda_{1}$ ) survives [Figs. 5(e) and 5(f)]. Asymptotically we find the behavior predicted by Pierrehumbert [15]: that the contrast (2) of the scalar density decreases without change of the spatial structure. A similar picture is also observed for the other sets of parameters $\left(U_{1}=5\right.$ and $\left.U_{2}=3\right)$ corresponding to Fig. 1(a).

Thus, during a large intermediate time range the two largest eigenvalues $\lambda_{1}$ and $\lambda_{2}$ together determine the decay rate of the pattern contrast (2). In order to describe this process quantitatively we expand an initial scalar density $\phi(x, y, 0)$ in the eigenbasis $\left\{\varphi_{i}\right\}$ of the FP operator (5) and obtain, after a few initial iterations,

$$
\phi(x, y, n)=\sum_{i} a_{i} \lambda_{i}^{n} \varphi_{i}(x, y)
$$

where $a_{i}$ are some constants. Neglecting small contributions connected with small eigenvalue terms $\lambda_{3}, \lambda_{4}$, etc., we can approximate $\phi(x, y, n)$ using only three eigenfunctions corresponding to the maximal eigenvalues $\lambda_{0}, \lambda_{1}$, and $\lambda_{2}$ :

$$
\phi(x, y, n) \approx a_{0} \varphi_{0}(x, y)+a_{1} \lambda_{1}^{n} \varphi_{1}(x, y)+a_{2} \lambda_{2}^{n} \varphi_{2}(x, y) .
$$

Finally, for the pattern contrast (2) we have

$$
\delta \phi^{2}(n) \sim b_{1} \lambda_{1}^{2 n}+b_{2} \lambda_{2}^{2 n} .
$$

In order to check this expression the continuous system (1) and (3) was numerically integrated for the parameters $U_{1}$ $=12$ and $U_{2}=1.14$. For the behavior after full periods we can also iterate the FP operator (5), which we did for the parameter values $U_{1}=5$ and $U_{2}=3$. The decay of the pattern contrast $\delta \phi$ versus time for the two cases is shown in Fig. 6. The agreement between eigenvalues of the FP operator (5) and the decay rates is good: the figure caption gives values for the eigenvalues $\lambda_{i}^{\prime}$ obtained by a direct fit to the data using the functional form (6) that are in good agreement with the eigenvalues $\lambda_{i}$ obtained from the Floquet analysis (see Table I). Note that because of the superposition of at least two exponentials the decay of contrast is not a simple exponential, as has been also found in the experiments [11].

In connection with mixed phase spaces one often expects nonexponential, power-law-like behavior [28]. Plots of the contrast on a doubly logarithmic scale in the insets in Fig. 6 show that for the case $U_{1}=5$ and $U_{2}=3$ [Fig. 6(a)] there is a time interval where a power-law approximation appears reasonable, but there is no such interval for the case $U_{1}=12$ and $U_{2}=1.14$ [Fig. 6(b)].

We have considered the simplest case where only two largest smaller than unity eigenvalues are close. Of course, situations where more than two eigenmodes participate in the pattern competition process are possible as well. We also note that in the cases studied here the time interval over which the nonexponential decay of the contrast is observed depends strongly on initial conditions. This reflects a strong variation of the coefficients $a_{i}$ with initial conditions, as one might expect. Specifically, there are initial conditions where the amplitude of the second mode is small, so that after 10-20 iterations only one mode survives and the further decay is a simple exponential with $\lambda_{1}$.

Of course, effects connected with the presence of nearby eigenvalues are strongest in cases of perfect degeneracy 
(where they can pick up a linear times exponential contribution; see [29]). But they also persist in a neighborhood in parameter space near these exact degeneracies: if the eigenvalues in each domain are widely separated and if the eigenvalues change slowly with parameters, the condition of overlap-i.e., the distance between eigenvalues smaller than the gap to the next one-can be satisfied in a significant range of parameters.

The spatial form of the slowest eigenmodes reflects the structure of the phase portrait of the flow and its dynamics. These modes are preferentially located in domains where the mixing rate in the system is relatively slow-e.g., in or near elliptic islands or near boundaries of chaotic regions.

In conclusion, we have shown that persistent spatial structures recently observed in experiments [11-13] can be modeled and studied using linear Frobenius-Perron operators. The description in terms of eigenmodes of this operator allows us to describe evolution at large times, where the slowest eigenmodes determine the form of the spatial structures. The coexistence of eigenmodes with relatively close eigenvalues gives rise to nonexponential decay and competition between structures over some time interval. We have demonstrated that such eigenmodes can be supported by dynamical objects of a different nature in the deterministic flow structure: large elliptic islands or invariant (or quasiinvariant) chaotic regions and their boundaries. This kind of near degeneracy has to be expected in systems with discrete symmetries which multiply invariant sets, but as the examples show, symmetries are no prerequisite. Another example where such trapping can occur is in the presence of walls, as a recent study [30] shows.

This work was partially supported by the EU within the Research and Training Network "Non-ideal turbulence," Grant No. HPRN-CT-2000-00162, and the DFG.
[1] H. Aref, J. Fluid Mech. 143, 1 (1984).

[2] H. Aref, Phys. Fluids 14, 1315 (2002); 14, 2395 (2002).

[3] J. M. Ottino, The Kinematics of Mixing: Stretching, Chaos, and Transport (Cambridge University Press, Cambridge, England, 1989).

[4] I. Mezić and S. Wiggins, J. Nonlinear Sci. 4, 157 (1994).

[5] I. Mezić, J. Fluid Mech. 431, 347 (2001).

[6] V. Rom-Kedar and A. Poje, Phys. Fluids 11, 2044 (1999).

[7] H. A. Stone and S. Kim, AIChE J. 47, 1250 (2001).

[8] A. D. Stroock, S. K. W. Dertinger, A. Ajdari, I. Mezic, H. A. Stone, and G. M. Whitesides, Science 295, 647 (2002); S. Balasuriya, Phys. Fluids 17, 118103 (2005).

[9] T. H. Solomon and I. Mezić, Nature (London) 425, 376 (2003).

[10] Transport and Mixing at the Microscale [Philos. Trans. R. Soc. London, Ser. A 362, 1818 (2004)].

[11] D. Rothstein, E. Henry, and J. P. Gollub, Nature (London) 401, 770 (1999).

[12] J. Deval and P. Tabeling, Phys. World 13(1), 22 (2000).

[13] G. A. Voth, G. Haller, and J. P. Gollub, Phys. Rev. Lett. 88, 254501 (2002).

[14] P. E. Arratia and J. P. Gollub, Phys. Rev. Lett. 96, 024501 (2002).

[15] R. T. Pierrehumbert, Chaos, Solitons Fractals 4, 1091 (1994).

[16] S. Childress and A. D. Gilbert, Stretch, Twist, Fold: The Fast Dynamo (Springer-Verlag, Berlin, 1995).

[17] D. R. Fereday, P. H. Haynes, A. Wonhas, and J. C. Vassilicos,
Phys. Rev. E 65, 035301(R) (2002).

[18] A. Pikovsky and O. Popovych, Europhys. Lett. 61, 625 (2003).

[19] J.-L. Thiffeault and S. Childress, Chaos 13, 502 (2003).

[20] W. Liu and G. Heller, Physica D 188, 1 (2004).

[21] M. Giona, A. Adrover, S. Cerbelli, and V. Vitacolonna, Phys. Rev. Lett. 92, 114101 (2004).

[22] W. Liu, Phys. Rev. E 72, 016312 (2005).

[23] Y.-K. Tsang, T. M. Antonsen, and E. Ott, Phys. Rev. E 71, 066301 (2005).

[24] B. Jüttner, D. Marteau, P. Tabeling, and A. Thess, Phys. Rev. E 55, 5479 (1997); B. Eckhardt, E. Hascoet, and W. Braun, in Proceedings of the IUTAM Symposium on Nonlinear Stochastic Dynamics, edited by N. Sri Namachchavaya and Y. K. Lin (Kluwer, Amsterdam, 2003), pp. 415-424; B. Eckhardt and E. Hascoet, Phys. Rev. E 72, 037301 (2005).

[25] T. M. Antonsen, Z. Fan, E. Ott, and E. Garcia-Lopes, Phys. Fluids 8, 3094 (1996).

[26] M. Khodas and S. Fishman, Phys. Rev. Lett. 84, 2837 (2000); M. Khodas, S. Fishman, and O. Agam, Phys. Rev. E 62, 4769 (2000).

[27] B. Eckhardt and D. Yao, Physica D 65, 100 (1993).

[28] B. V. Chirikov and D. L. Shepelyansky, Physica D 13, 395 (1984).

[29] B. Eckhardt and S. Grossmann, Phys. Rev. E 50, 4571 (1994).

[30] E. Gouillart, N. Kuncio, O. Dauchot, B. Dubrulle, S. Roux, and J.-L. Thiffeault, e-print cond-mat/0612557. 